\title{
The Star Formation History of the GRB 050730 Host Galaxy
}

\author{
Francesco Calura ${ }^{1}$ \\ ${ }^{1}$ Dipartimento di Astronomia, Universita' di Trieste, via G. B. Tiepolo 11, 34131 \\ TRIESTE - ITALY \\ email: fcalura@oats.inaf.it
}

\begin{abstract}
The long GRB 050730 observed at redshift $z \sim 4$ allowed the determination of the elemental abundances for a set of different chemical elements. We use detailed chemical evolution models taking into account also dust production to constrain the star formation history of the host galaxy of this long GRB. For the host galaxy of GRB 050730, we derive also some dustrelated quantities and the specific star formation rate, namely the star formation rate per unit stellar mass. Finally, we compare the properties of the GRB host galaxy with those of Quasar Damped Lyman Alpha absorbers.
\end{abstract}

Keywords. Gamma rays: bursts, Galaxies: high-redshift, Galaxies: abundances, ISM: general

\section{Introduction}

Gamma-ray bursts (GRBs) afterglows provide insight into the interstellar medium (ISM) of galaxies during the earliest stages of their evolution. In several cases, thanks to the GRB afterglows it has been possible to study the dust content, the star formation rates and the stellar mass of the GRB host (Bloom et al. 1998; Savaglio et al. 2008). In a few cases, the determination of their chemical abundance pattern has been possible (Savaglio et al. 2003; Vreeswijk et al. (2004); Prochaska et al. 2007).

Chen et al. (2005) reported on the chemical abundances for the damped Lyman Alpha system (DLA) associated with the host galaxy of GRB 050730. Their analysis showed that this gas was metal poor with modest depletion. These results were subsequently expanded and tabulated by Prochaska et al. (2007) (hereafter P07).

In this paper, we aim at determining the star formation history of a GRB host galaxy by studying the chemical abundances measured in the afterglow spectrum of GRB 050730 . For this purpose, we use a detailed chemical evolution model. Our aim is to constrain the star formation rate and the age of the host galaxy of GRB 050730, and possibly to expand our study to other systems. The plan of this paper is as follows. In Section 2, we briefly introduce the chemical evolution model. In Section 3, we present and discuss our main results.

\section{Chemical evolution models including dust}

The chemical evolution model used here to derive the star formation history of the GRB 050730 host galaxy is similar to that developed by Bradamante et al. (1998) for dwarf irregular galaxies. Since some chemical species studied in this paper are refractory $(\mathrm{Fe}, \mathrm{Ni})$, in the chemical evolution model we include also a treatment of dust production and destruction, based on the work by Calura, Pipino \& Matteucci (2008, hereafter CPM08). 
We use the model for dwarf irregular galaxies since several observational investigations provided strong evidence that most of the GRBs originate in gas rich, star-forming subluminous $\left(L<L_{*}\right)$ galaxies with relatively low metallicities $\left(Z<Z_{\odot}\right)$ (Bloom et al. 1998; Prochaska et al. 2004). The dwarf galaxy is assumed to form by means of a continuous infall of pristine gas until a mass $M_{t o t}$ is accumulated. The evolution of dwarf irregular galaxies is characterized by a continuous star formation history, characterized by a low star formation efficiency $\left(\nu \leqslant 0.1 G y r^{-1}\right)$. The star formation rate $\psi(t)$ is directly proportional the gas fraction $G(t)$ at the time $t$, according to the Schmidt law:

$$
\psi(t)=\nu G(t)
$$

Supernovae (SNe) Ia and II are responsible for the onset of a galactic wind, when the thermal energy of the ISM exceeds its binding energy, which is related to the presence of a dark matter halo in which the galaxy is embedded (for more details, see Bradamante et al. 1998, Lanfranchi \& Matteucci 2003). The binding energy of the gas is influenced by assumptions concerning the presence and distribution of dark matter (Matteucci 1992). A diffuse $\left(R_{e} / R_{d}=0.1\right.$, where $R_{e}$ is the effective radius of the galaxy and $R_{d}$ is the radius of the dark matter core) and massive $\left(M_{\text {dark }} / M_{\text {Lum }}=10\right)$ dark halo has been assumed for each galaxy. The time at which the wind develops depends on the assumed star formation efficiency. In general, the higher the star formation (SF) efficiency, the earlier the wind develops. The models used throughout this paper are characterized by very low star formation efficiencies and by a young age, much lower than the times of the onset of the galactic winds. For these reasons, the galactic winds have no effect on the main results obtained in this paper.

Chemical enrichment from various types of stars is properly taken into account. The stellar yields are mainly from Woosley \& Weaver (1995) for massive stars, from Meynet \& Maeder (2002) for low and intermediate mass stars (LIMS) and from Iwamoto et al. (1999) for Type Ia SNe.

We assume a Salpeter initial mass function (IMF). We assume a cosmological model characterized by $\Omega_{m}=0.3, \Omega_{\Lambda}=0.7$ and a Hubble constant $H_{0}=70 \mathrm{~km} \mathrm{~s}^{-1} \mathrm{Mpc}^{-1}$.

\subsection{Dust production and destruction}

Two elements studied in these paper are refractory: Ni and Fe. To model their gas phase abundances, we need to take into account dust production and destruction.

The model for dust evolution used in this paper is described in detail in CPM08. Here, we summarize its main features. For the refractory chemical element labeled $i$, a fraction $\delta_{i}^{S W}, \delta_{i}^{I a}$, and $\delta_{i}^{I I}$ is incorporated in dust grains by low and intermediate mass stars, type Ia SNe, and type II SNe, respectively. These quantities are the dust condensation efficiencies of the element $i$ in various stellar objects. Here we assume $\delta_{i}^{S W}=\delta_{i}^{I a}=\delta_{i}^{I I} \equiv$ $\delta_{i}=0.1$. This choice is motivated by recent mid-infrared observations of one supernova (Sugerman et al. 2003), which provided an upper limit of $\delta_{i}^{I I} \leqslant 0.12$. The value assumed here is further supported by theoretical studies of the local dust cycle (Edmunds 2001).

Dust grains are usually destroyed by the propagation of SN shock waves in the warm/ionised interstellar medium (Jones et al. 1994). If $G_{d u s t, i}$ is the fraction of the element $i$ locked into dust and $G$ is the gas fraction, the destruction rate is calculated as $G_{d u s t, i} / \tau_{d e s t r}$, where $\tau_{\text {destr }}$ is the dust destruction timescale, calculated as:

$$
\tau_{\text {destr }}=\left(\epsilon M_{S N R}\right)^{-1} \frac{G}{R_{S N}} .
$$

(McKee 1989). $M_{S N R}=1300 M_{\odot}$ is the mass of the interstellar gas swept up by the SN remnant. $R_{S N}$ is the total SNe rate, i.e. the sum of the rates of Type Ia and Type II SNe. 
Unless otherwise specified, we assume that no dust accretion, occurring mainly in cold molecular clouds, is taking place in the GRB host galaxy. Our choice is motivated by the fact that very little molecular $\mathrm{H}$ is observed in local dwarfs, with molecular-to-atomic gas fractions of $\sim 10 \%$ or lower (Clayton et al. 1996). Our assumption is further supported by the lack of $\mathrm{H}_{2}$ absorption lines observed in the spectra of GRB afterglows (Whalen et al. 2008). In the presence of intense SF, molecular clouds are likely to rapidly dissolve, allowing very little dust accretion to occur.

\subsection{Results and discussion}

The abundance ratios between two elements formed on different timescales can be used as "cosmic clocks" and provide us with information on the roles of LIMS and SNe in the enrichment process (Matteucci 2001). In particular, the study of abundance ratios such as $[\alpha / \mathrm{Fe}]$ and $[\mathrm{N} / \alpha] \dagger$ is quite helpful, since the $\alpha$-elements $(\mathrm{O}, \mathrm{S}, \mathrm{Mg})$ are produced on short timescales by Type II SNe, whereas the Fe-peak elements and nitrogen are produced on long timescales by Type Ia SNe and low and intermediate-mass stars, respectively. As shown by Dessauges-Zavadsky at al. (2004; 2007, hereafter D07), the simultaneous study of the abundance ratios between different elements as functions of a metallicity tracer (such as $[\mathrm{S} / \mathrm{H}]$ or $[\mathrm{Fe} / \mathrm{H}]$ ) may be used to constrain the star formation history of a given system, whereas the study of the abundance ratios versus redshift can be used to constrain the age of the system.

$\dagger$ All the abundances between two different elements $\mathrm{X}$ and $\mathrm{Y}$ are expressed as $[X / Y]=\log (X / Y)-\log (X / Y)_{\odot}$, where $(X / Y)$ and $(X / Y) \odot$ are the ratios between the mass fractions of $\mathrm{X}$ and $\mathrm{Y}$ in the ISM and in the sun, respectively. We use the set of solar abundances as determined by Grevesse et al. (2007).
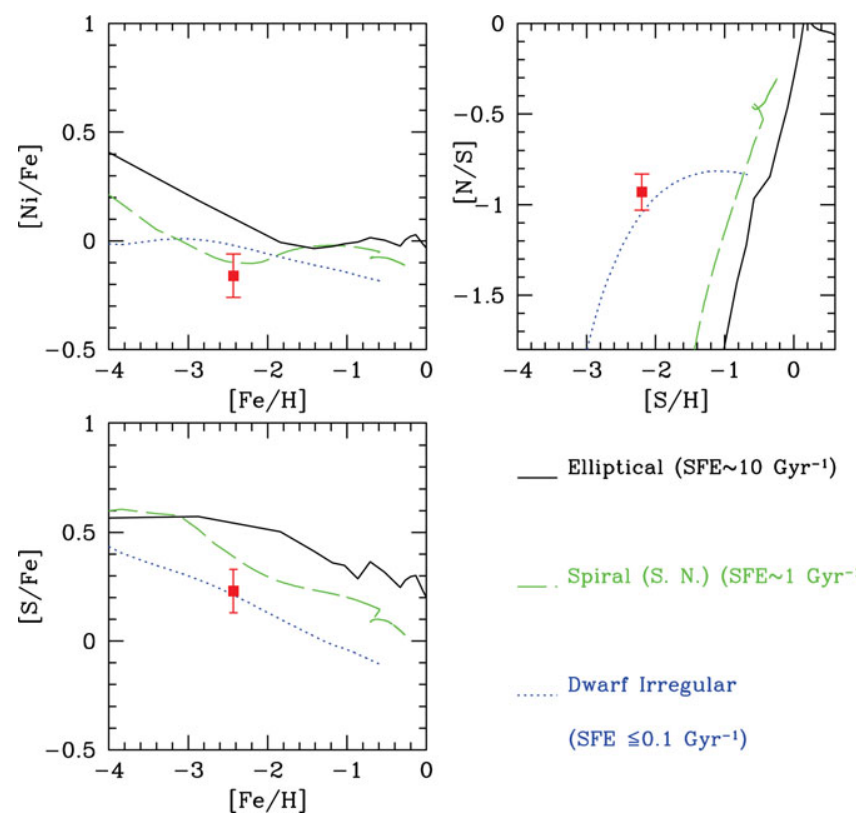

_ Elliptical (SFE 10 $\left.\mathrm{Gyr}^{-1}\right)$

_. Spiral (S. N.) (SFE 1 $\left.\mathrm{Gyr}^{-1}\right)$

...... Dwarf Irregular

$\left(\mathrm{SFE} \leqq 0.1 \mathrm{Gyr}^{-1}\right)$

Figure 1. Observed abundance ratios versus metallicity for the host galaxy of GRB 050730 as reported by P07 (solid squares with error bars). The solid line, dashed line and dotted line are predictions computed by means of a chemical evolution model for an elliptical galaxy, a spiral galaxy and a dwarf irregular galaxy, respectively, and without including dust depletion (see text for further details). 

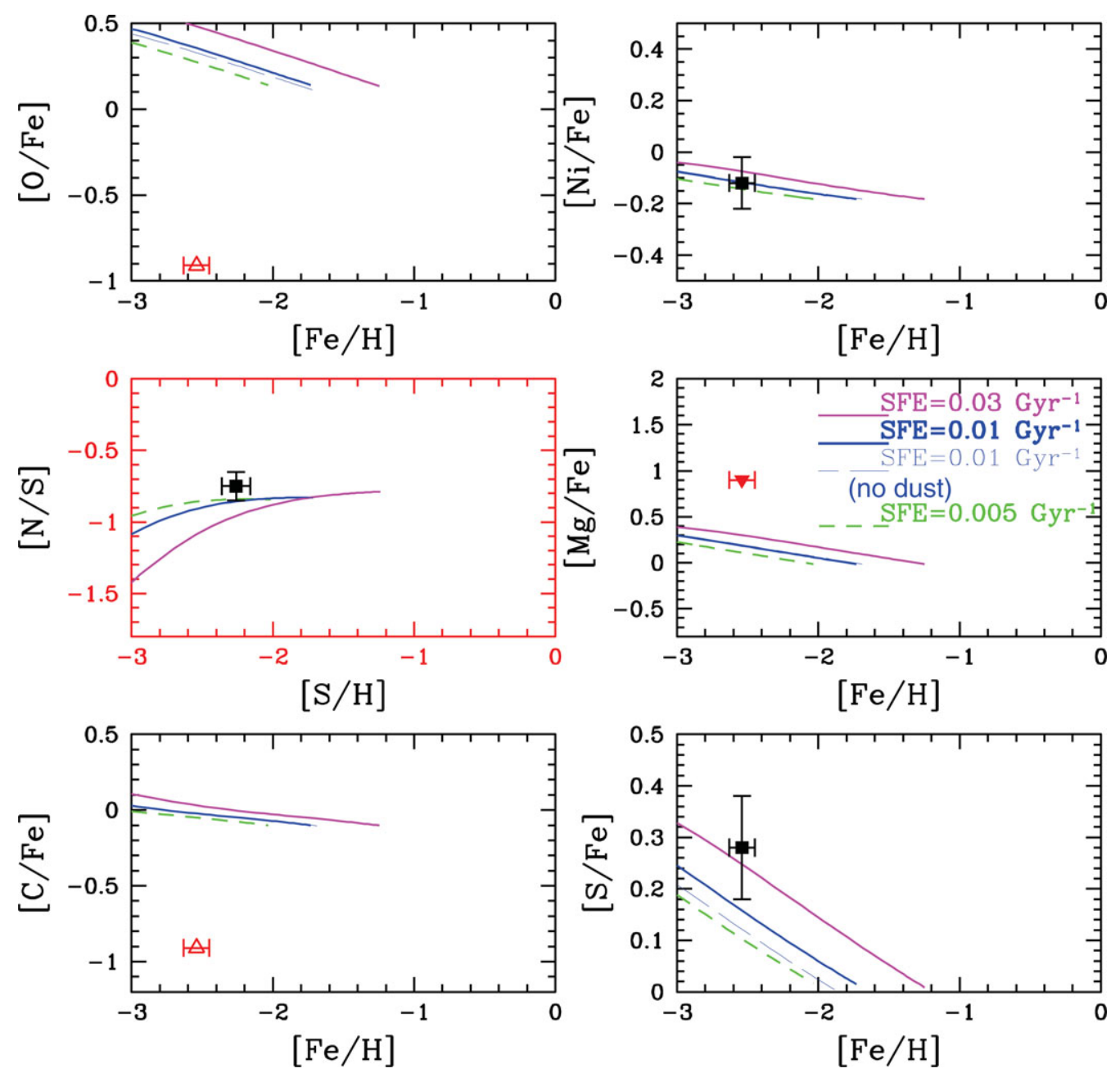

Figure 2. Observed abundance ratios versus metallicity for the host galaxy of GRB 050730 as derived by P07 (solid squares with error bars). The triangles stand for upper or lower limits. The thick lines represent models with increasing SF efficiency, with the lowest curves having the lowest SF efficiency values. The thin dashed lines do not include dust depletion. The red box $(\mathrm{N} / \mathrm{S}$ vs $\mathrm{S} / \mathrm{H})$ is used for abundance ratios between non-refractory elements.

In Fig. 1, we show the predicted evolution of $[\mathrm{N} / \mathrm{Fe}],[\mathrm{Ni} / \mathrm{Fe}]$ and $[\mathrm{S} / \mathrm{Fe}]$ as a function of various metallicity tracers, such as $[\mathrm{Fe} / \mathrm{H}]$ or $[\mathrm{S} / \mathrm{H}]$, for various star formation histories describing galaxies of different morphological types: an elliptical galaxy (solid lines), a spiral galaxy (more precisely, a model for the Solar Neigbourhood, dashed lines, see CPM08 for a detailed model description) and a dwarf irregular galaxy (dotted lines), compared with the values observed for GRB 050730 DLA (solid squares with error bars). Fig 1 shows clearly that the most likely progenitor for the host galaxy of GRB 050730 is a dwarf irregular galaxy, and how the elliptical and spiral model appear indadequate to describe the abundance pattern observed by P07 in the GRB 050730 host. In the following, we will use the dwarf irregular galaxy model to constrain the parameters of the SF history and the age of the host galaxy GRB 050730.

The next step is to constrain the main star formation history parameter of the host galaxy of GRB 050730, i.e. its star formation efficiency $\nu$. We search for the model which 

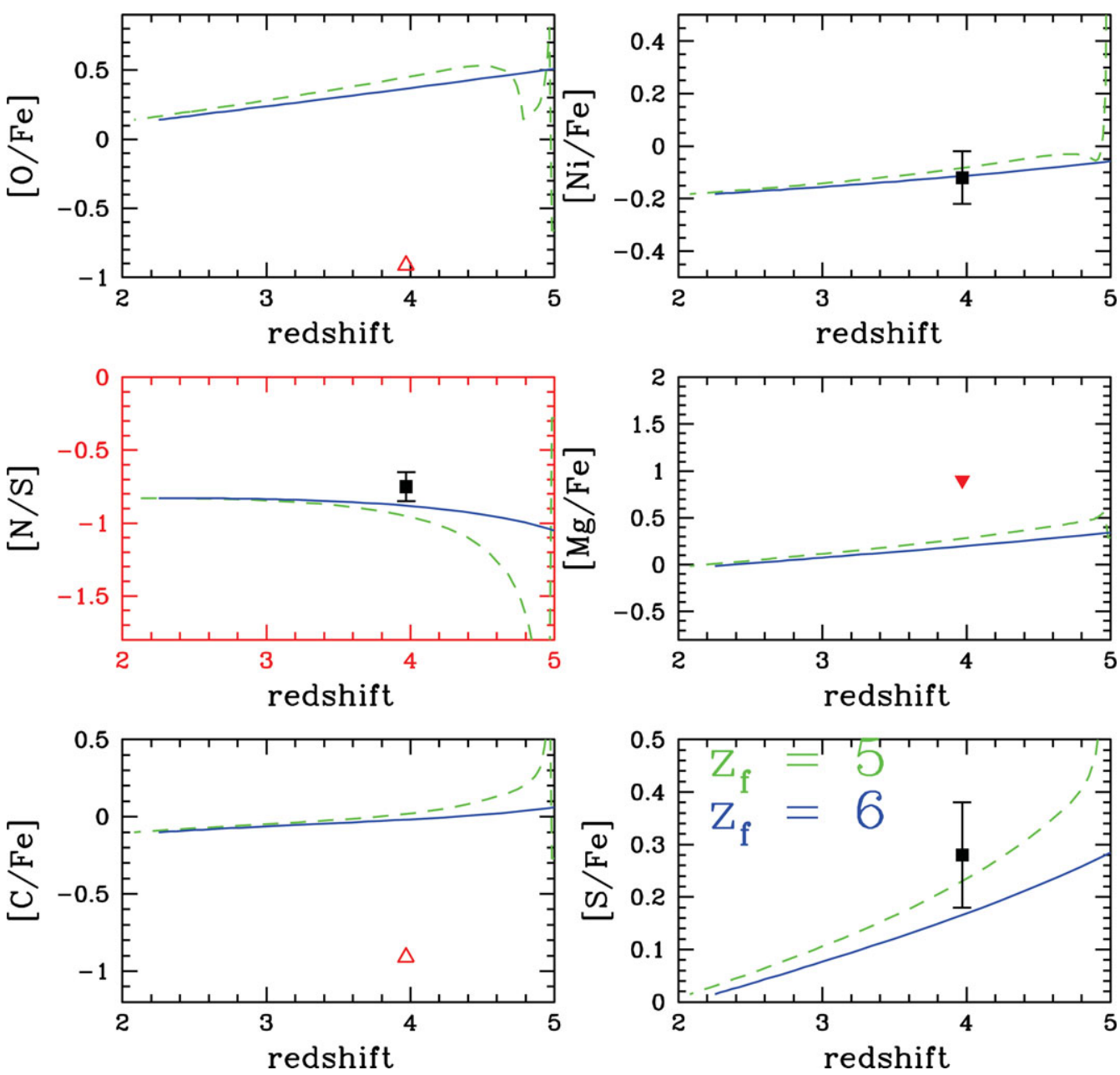

Figure 3. Observed abundance ratios versus redshift for the host galaxy of GRB 050730 as derived by P07 (solid squares with error bars). The triangles stand for upper or lower limits. The solid (dashed) lines represent a redshift of formation $z_{f}=6\left(z_{f}=5\right)$. The thin dashed lines do not include dust depletion. The red box (N/S vs redshift) is used for abundance ratios between non-refractory elements.

best reproduces the observed abundances. In figure 2, we show the predicted evolution of several abundance ratios for various models, characterized by different SF efficiencies. The model which best reproduces the observed abundances is that characterized by a SF efficiency $\nu=0.01 \mathrm{Gyr}^{-1}$.

Once we have determined the star formation efficiency of the best model, the following step is to constrain the age of the GRB host galaxy. To perform this task, we study the abundance ratios vs redshift (Fig. 3). The abundances are best reproduced at an age of $\sim 0.4 \mathrm{Gyr}$, corresponding to a redshift of formation $z_{f} \sim 5$. At this age, the specific star formation rate is $\sim 5 \mathrm{Gyr}^{-1}$, the dust-to-gas ratio is $\sim 10^{-6}$, in agreement with the upper limit of $8 \cdot 10^{-6}$ as derived observationally by P07. The dust-to-metals ratio is 0.04 , roughly consistent with the upper limit of 0.1 measured by P07. 


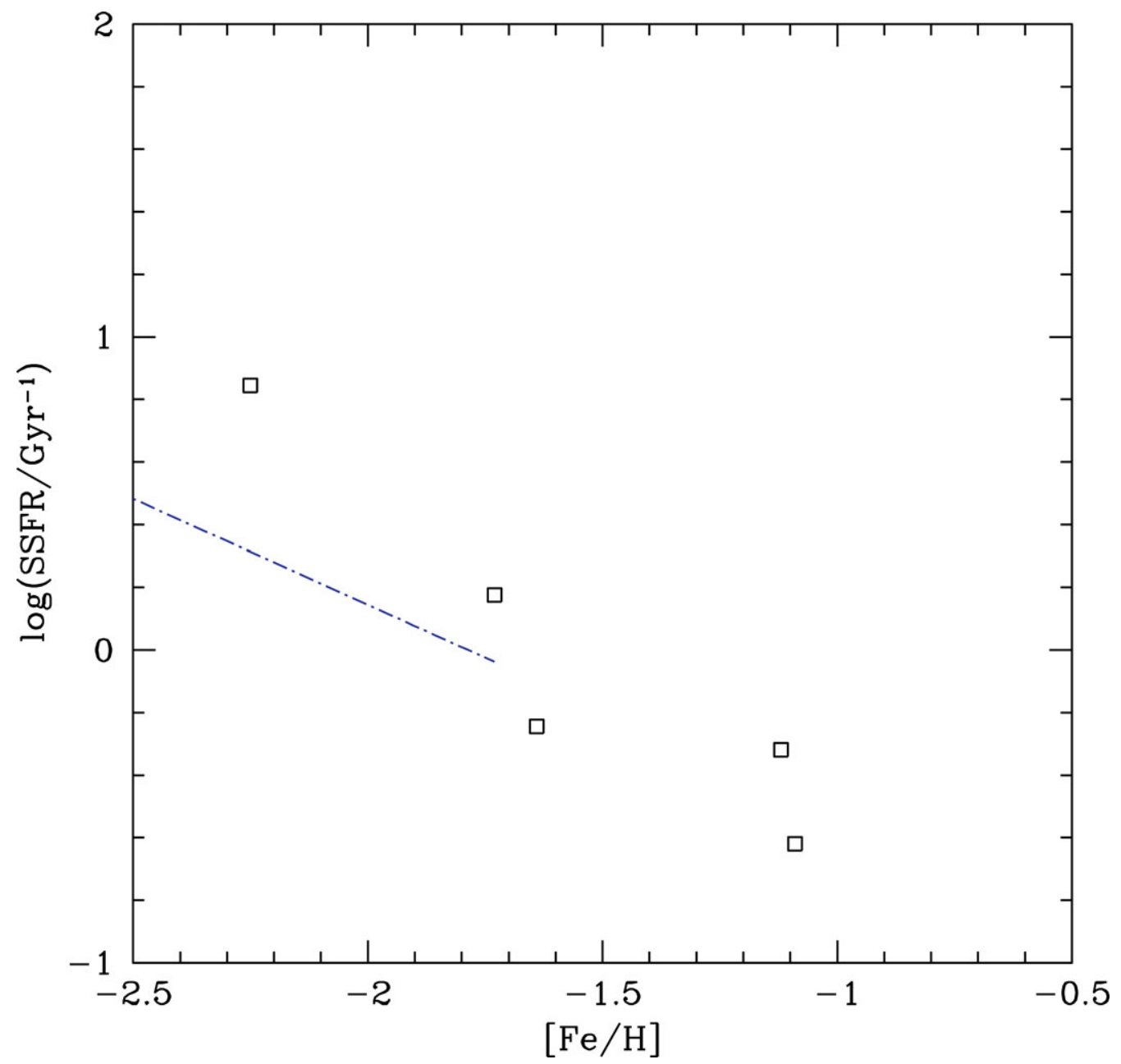

Figure 4. Predicted evolution of the SSFR vs $[\mathrm{Fe} / \mathrm{H}]$ for the best model for the host galaxy of GRB 050730. The open squares are the values determined for 5 QSO DLAs in the sample by D07.

Finally, in Fig. 4, we compare the predicted SSFR vs $[\mathrm{Fe} / \mathrm{H}]$ obtained for the best model for the host galaxy of GRB 050730, with SSFR derived for a sample of QSO DLAs by D07. The evolution of the SSFR of the GRB host is compatible with the values found for QSO DLAs, possibly indicating similar chemical evolution paths. However, this may be the result of a coincidence, since in principle GRB DLAs are expected to represent denser and more metal-enriched galactic regions than QSO DLAs. By extending the method presented here to other systems, it will be possible to shed light on possible analogies and differences between QSO and GRB DLAs.

\section{Acknowledgements}

It is a pleasure to thank my collaborators, i.e. Jason Prochaska, Mirka DessaugesZavasky and Francesca Matteucci. I acknowledge financial contribution from contract ASI-INAF I/016/07/0. 


\section{References}

Bloom, J. S.; Djorgovski, S. G.; Kulkarni, S. R.; Frail, D. A., 1998, ApJ, 507, L25

Bradamante, F., Matteucci, F., D'Ercole, A. 1998, A\&A,337, 338

Calura, F.; Pipino, A.; Matteucci, F., 2008, A\&A, 479, 669 (CPM08)

Clayton, Geoffrey C., Green, J., Wolff, Michael J., Zellner, Nicolle E. B., Code, A. D., Davidsen, Arthur F., WUPPE Science Team, HUT Science Team, 1996, ApJ, 460313

Dessauges-Zavadsky, M.; Calura, F.; Prochaska, J. X.; D'Odorico, S.; Matteucci, F., 2004, A\&A, 416, 79

Dessauges-Zavadsky, M.; Calura, F.; Prochaska, J. X.; D’Odorico, S.; Matteucci, F., 2007, A\&A, $470,431(\mathrm{D} 07)$

Edmunds, M. G., 2001, MNRAS, 328, 223

Grevesse, N.; Asplund, M.; Sauval, A. J., 2007, SSRv, 130, 105

Jones, A. P., Tielens, A. G. G. M., Hollenbach, D. J., McKee, C. F., 1994, ApJ, 433, 797

Iwamoto, K.; Brachwitz, F.; Nomoto, K.; Kishimoto, N.; Umeda, H.; Hix, W. R.; Thielemann, F.-K., 1999, ApJS, 125, 439

Lanfranchi, G., Matteucci, F., 2003, MNRAS, 345, 71

Matteucci, F., 1992, ApJ, 397, 32

Matteucci, F., 2001, The chemical evolution of the Galaxy, Astrophysics and space science library, Volume 253, Dordrecht: Kluwer Academic Publishers

McKee C. F., 1989, in Allamandola L. J., Tielens A. G. G. M., eds, Interstellar Dust, Proc. IAU Symposium 135. Kluwer, Dordrecht, p. 431

Meynet, G., Maeder, A., 2002, A\&A, 381, L25

Prochaska, J. X.; Bloom, J. S.; Chen, H.-W.; Hurley, K. C.; Melbourne, J.; Dressler, A.; Graham, J. R.; Osip, D. J.; Vacca, W. D., 2004, ApJ, 611, 200

Prochaska, J. X.; Chen, H.-W.; Dessauges-Zavadsky, M.; Bloom, J. S., 2007, ApJ, 666, 267

Savaglio, S., Fall, S. M., Fiore, F., 2003, ApJ, 585, 638

Savaglio, S., Glazebrook, K., Le Borgne, D., 2008, ApJ, submitted , arXiv0803.271

Sugerman, B. E. K., et al., 2006, Science, 313, 196

Vreeswijk, P. M., et al., 2004, A\&A, 419, 927

Whalen, D., Prochaska, J. X., Heger, A., Tumlinson, J., 2008, ApJ, submitted

Woosley, S.E., Weaver, T.A., 1995, ApJS, 101, 181 